\title{
Implementating the optimal operation point of Induction Generator using LabVIEW
}

\author{
Marshiana.D, Geetha.V, Krishnamoorthy.N.R, Rohith.V, Dinesbalaji.K.P \\ \{d.marshiana@gmail.com, geethasendray28@gmail.com,moorthy26.82@gmail.com\} \\ Sathyabama Institute of Science and Technology,Chennai-119
}

\begin{abstract}
Wind system permits adjustable operation for numerous wind generators which are parallel coupled for the production of power by dispensing with the necessity of voltage requirement. The operational operating point is observed for the induction generator for the production of power. A model prescient control calculation was developed to check the capacity of the power generated in the micro grid. This offers an enhanced transient response regarding the development and control of the working conditions of inverters. The analysis of the system is made by measuring the speed of the windmill and the outcomes are obtained using LabVIEW.
\end{abstract}

Keywords: Wind system, LabVIEW, Induction Generator,Operational operating point.

\section{Introduction}

The Earth needs energy from the sun and it is haphazardly heated, the energy from the sun is more in equator when compared with the poles. The dry soil in earth gets heated up hurriedly than the area covered by water. The change in heating of earth's atmosphere provides a universal atmospheric convection at the stratosphere of the Earth's surface that acts as a virtual ceiling. Ultimately, the wind energy is produced because of heat diffusion in the atmosphere and the surface of the earth. The major part of the energy stored due to these wind motion is found at high altitudes where unremitting speed of wind is over $160 \mathrm{~km} / \mathrm{h}(99 \mathrm{mph})$.

The statistical report says that the amount of wind power available in our country is utilized more than other power sources. The most widespread studies are made were done related to the usage of power as of 2005 originate that the potential use of wind power is 72 TW, comparable to 54,000 MTOE. The wind turbine produces energy with the frequency having a meticulous locality of wind speed. The optimal data is observed to fit inside the probability distribution function. The wind distribution changes in different area based on the atmospheric conditions. The Weibull technique is used for the calculation which relates the wind speed in hourly basics at several locations and is generally closed to 2 . The significance of the wind energy from an exacting turbine or wind farm is that, it cannot have a reliable result when compared with the fuel-fired power plants.

In general the power generation from wind farm is based on the Induction generators which require reactive power for excitation. The transmission of power generated provides different behavior based on the types of wind turbine. The new wind farm is to be developed based on extensive modeling which suits the characteristics of dynamic electromechanical 
system. This transmission system is mandatory to operators with guarantee optimal and predictable stable behavior when fault occurs. During fault condition the induction generators won't be able sustain the system voltage. The Doubly-fed machines normally have added advantageous for grid interconnection. The wind farm developed in rural areas needs operators for operating the will mill Transmission systems. The developers will supply a code for transmission grids to identify the specific information for interconnection. The wind mill transmission behavior includes, steadiness of frequency, power factor and dynamic operation during a system fault.

The process of identifying the operation at optimal level in an induction machine using Lab VIEW [1] developed virtually off-grid. Evaluation of induction generator on optimal results is partly achieved using EMTP - ATP [2]. The model for generators can be used for examine and estimate the control strategies using simulation for variable speed[3].The mathematical model assumed that a wind turbine and the rotor also solved the problem to obtain the optimal design for a small-scale turbine the can be formulated in terms of the pitch angle and working mode[4]. The financially viable analysis was studied for wind turbines of Johannesburg [5]. The direct maximum power point tracking (MPPT) scale is used in wind generator by permanent magnet synchronous machine (PMSM) [6]. Whereas control of smallscales wind turbines having Doubly Fed Induction Generator [7] and evaluates a small scale urban application [8]. Electric vehicles [9] are with electric power was considered to be environmentally friendly as a best transportation tool, which can efficiently reduce pollution and fossil fuel dependency.

\section{Methods and Methodology}

The methods used in wind turbine are to control the sliding mode(SM) using variable structure control. The wind energy produced from the wind turbine is achieved by using fast dynamical MPPT for the entire system. The performance analysis is based on the optimal technique achieved by using SM control. The block diagram representation of the optimal wind generator production is given in fig. 1

An Induction generator for the production of wind energy is designed based on the application. The advantage of the wind energy process at the dc grid is that only the voltage is to be maintained. The energy production is mainly controlled by the wind generator for parallel operation. In this the voltage is to be maintained without any error or disturbance and maintain at constant in phase with the frequency which allows the turbine to be switched ON or OFF. The initial status of power MOSFET is made simple with very small size so that the infinite current gain is produced for charging and discharging of capacitance in input device 


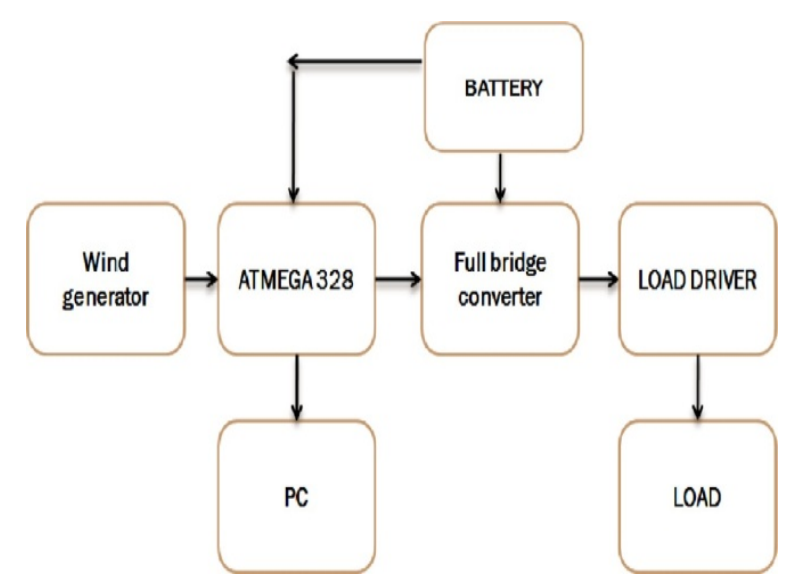

Fig.1 Block Diagram of Wind Generator

The energy produced by wind mill is applied to 8-bit microcontroller ATmega16 which is based on a low-power CMOS. It was developed by AVR enhanced RISC architecture. The powerful coding is executed within a single clock cycle. The ATmega16 achieve the impending 1 MIPS per MHz by allowing the system especially designed to optimize power consumption with respect to the processing speed. The ATmegal6 has major features which can be implemented for energy saving without the loss. The basic characteristics of this processor is having flash programmer with 16 Kbytes of In-System, where it can operate with Read-While-Write capabilities. The memory provides a major role in programming the processor having512 bytes EEPROM, and 1 Kbyte SRAM with JTAG interface for Boundary scan. A serial programmable USART is implemented a programmable Watchdog timer. It provides gain having optional differential input.

The ADC Noise Reduction mode is available in this circuit, which can stop the error occur in CPU and all I/O modules. During the. Switching process the noise developed in the $\mathrm{ADC}$ circuit can be minimized. A backup system is used that it can be withstand during any failure situations. Here the devices are made inactive while the wind mill is in ON state and the crystal Oscillator is operated in Standby mode. This condition provides low-power consumption and shares a very fast start-up. In Atmel ATmega16 microcontroller is a dominant device that gives a greater advantage such as flexibility and low cost problems for several embedded and control applications. The main characteristics of ATmega16 AVR system for program support and evaluation are program debugger/simulators, $\mathrm{C}$ compilers, incircuit emulators, macro assemblers,

Laboratory Virtual Instrument Engineering Workbench (Lab VIEW) is a virtual design tool for the development of newer techniques. This system-design platform can perform various operations depending on the need and its applications. The power generator from the wind mill is interfaced with block diagram of the circuit using data acquisition system. The library information provides deployment of data in running environment for analysis. The unique competence in Lab VIEW offers is real-time compilation with the voltage produce in windmill is executed by programming languages. The ability is that it can able to execute function blocks devoid of requiring improvement of a test case. This function can interact with design code instantaneously after program execution 


\section{Results and Discussion}

The wind power produced by the induction generator was optimized by interfacing using the LabVIEW software.

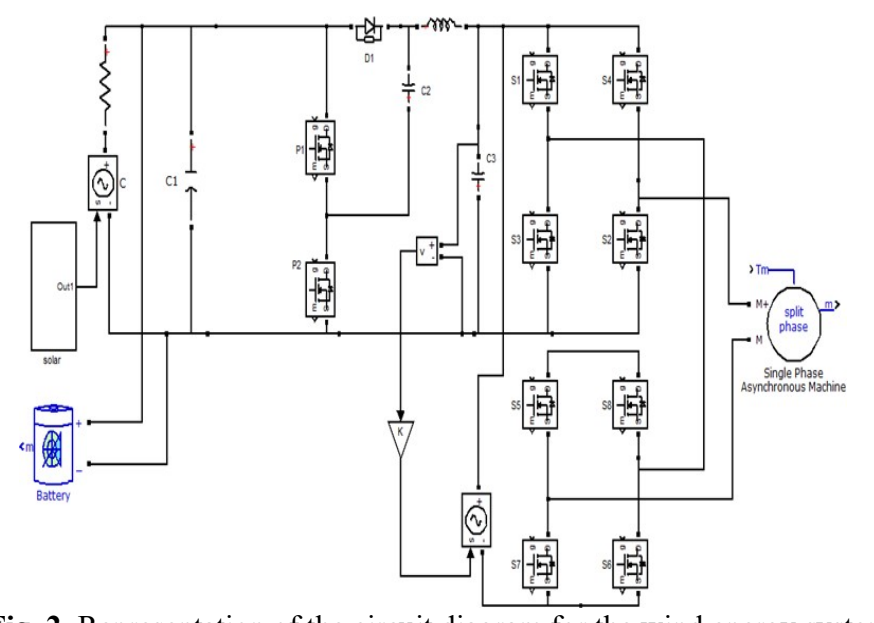

Fig. 2. Representation of the circuit diagram for the wind energy system

The power produced by wind mill is used for energy production and given to load. The excess enery is stored in batterythe windmill rotates along with the wind speed of $3 \mathrm{~V}$ wind voltage produces wind energy of $60 \mathrm{~V}$ and utilized.

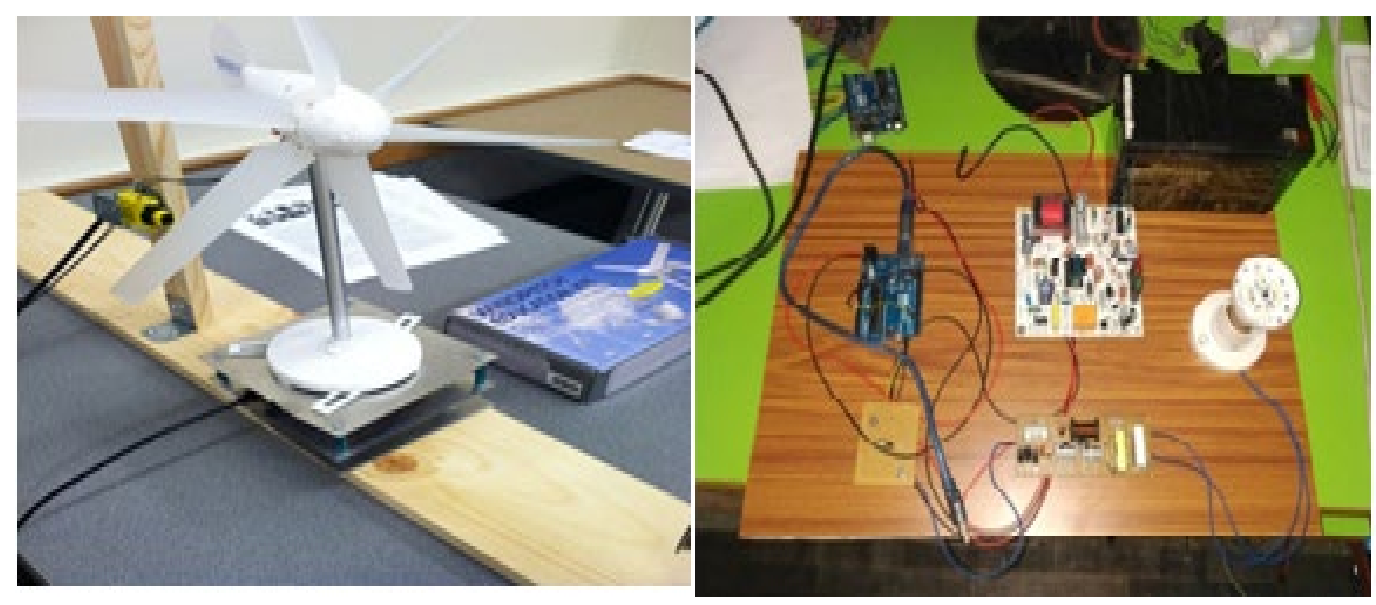

Fig 3. Prototype model of the wind energy system 

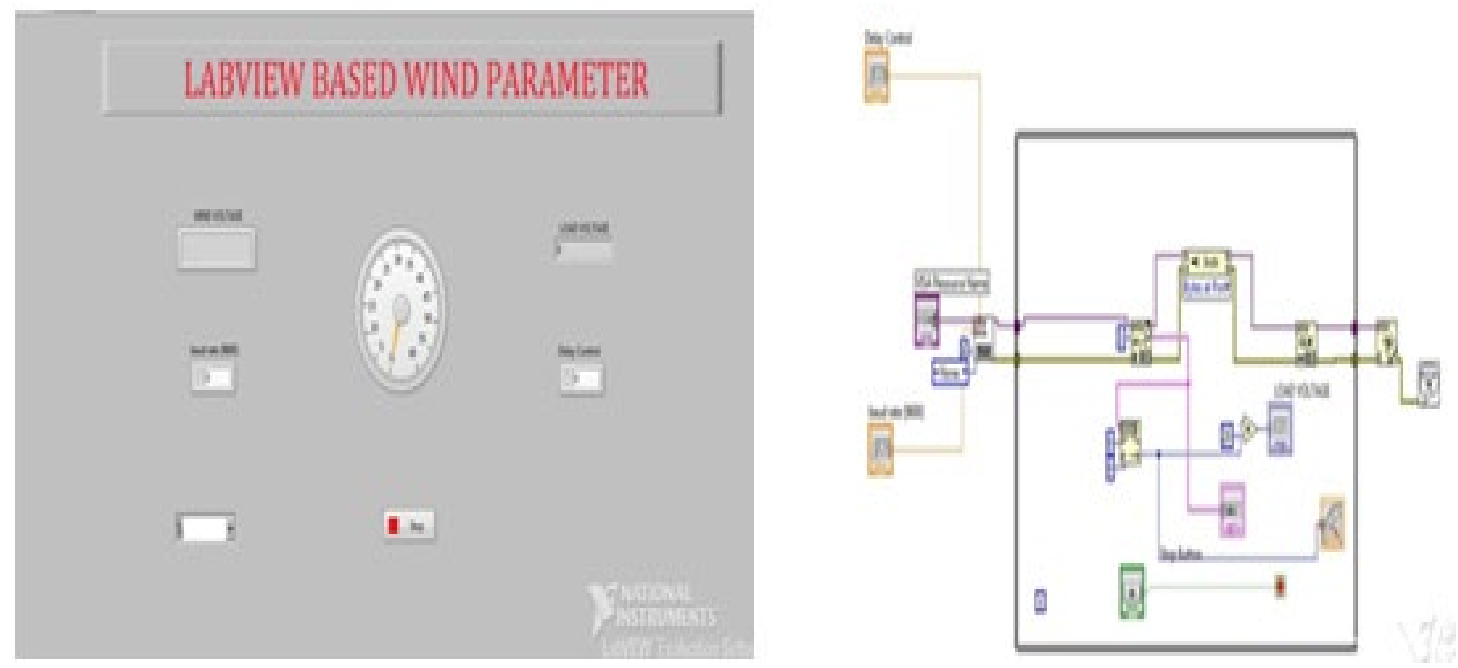

Fig 4: Front Panel and Block diagram of optimal operation of induction generator

\section{Conclusion}

Energy generated by the wind mill for increasing production of power. The developed prototype model for asynchronous machine is associated with small scale wind power. Induction generator is used for its simplicity. It requires only less maintenance and also has reduced acquisition price when compared with other generators. The output is interfaced using virtual software for analysis and increase in power production. The variable frequency based Induction machine produces more power with optimal characteristics is achieved. Thus the power plant can satisfy the need of energy using this wind mill so that the power utilization can be achieved in minimum cost and ideal size. 


\section{References}

[1] Divya, P.S., Lydia, M., Manoj, G. and Arumainayagam, S.D., Minimization of the Wind Turbine Cost of Energy through Probability Distribution Functions. In 2nd EAI International Conference on Big Data Innovation for Sustainable Cognitive Computing (pp. 25-32). Springer, Cham

[2] Joshuva, A., M. Arjun, R. Murugavel, V. A. Shridhar, GS Sriram Gangadhar, and S. S. Dhanush. "Predicting Wind Turbine Blade Fault Condition to Enhance Wind Energy Harvest Through Classification via Regression Classifier." In Advances in Smart Grid Technology, pp. 13-20. Springer, Singapore, 2020

[3] Arellano-Vázquez, Magali, Carlos Minutti-Martinez, and Marlene Zamora-Machado. "Automated Characterization and Prediction of Wind Conditions Using Gaussian Mixtures." In Mexican International Conference on Artificial Intelligence, pp. 158-168. Springer, Cham, 2020

[4] Nguyen, C. H., \& Huong, K. T. (2020). Experimental Modelling of Self-excited Responses of a Square Cylinder in Smooth Wind Flow. In ICSCEA 2019 (pp. 1101-1105). Springer, Singapore

[5] Zhang, Zhendong, Hui Qin, Jie Li, Yongqi Liu, Liqiang Yao, Yongqiang Wang, Chao Wang, Shaoqian Pei, and Jianzhong Zhou. "Short-term optimal operation of wind-solar-hydro hybrid system considering uncertainties." Energy Conversion and Management 205 (2020): 112405

[6] Caruso, Fabio, Mario Foglia, and Giulio Reina. "A Path Tracking Algorithm for an Autonomous Wind-Driven Robot." In The International Conference of IFToMM ITALY, pp. 542-550. Springer, Cham, 2020

[7] Chowdary VG, Sankar VU, Mathew D, Basha CH, Rani C. Hybrid Fuzzy Logic-Based MPPT for Wind Energy Conversion System

[8] InSoft Computing for Problem Solving 2020 (pp. 951-968). Springer, Singapore

[9] Colombo, L., M. L. Corradini, G. Ippoliti, and G. Orlando. "Pitch angle control of a wind turbine operating above the rated wind speed: A sliding mode control approach." ISA transactions 96 (2020): 95-102

[10] Cornejo-Bueno, Sara, Mihaela Ioana Chidean, Antonio J. Caamaño, Luís Prieto, and Sancho Salcedo-Sanz. "Comparing Traditional Methods of Complex Networks Construction in a Wind Farm Production Analysis Problem." In International Conference on Complex Networks and Their Applications, pp. 895-904. Springer, Cham, 2019. 